Accepted Manuscript

\title{
A STABILITY ANALYSIS OF THE COMPRESSIBLE BOUNDARY LAYER FLOW OVER INDENTED SURFACES
}

Jesús Garicano-Mena, Esteban Ferrer, Silvia Sanvido, Eusebio Valero

PII: S0045-7930(17)30369-9

DOI: 10.1016/j.compfluid.2017.10.011

Reference: CAF 3627

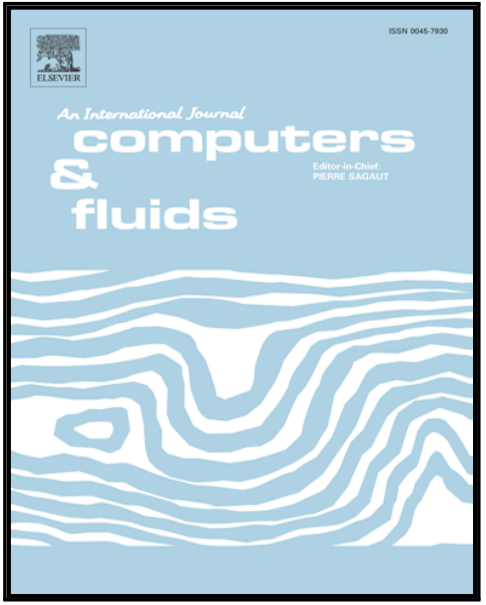

To appear in: Computers and Fluids

Received date: 16 January 2017

Revised date: 20 September 2017

Accepted date: 9 October 2017

Please cite this article as: Jesús Garicano-Mena, Esteban Ferrer, Silvia Sanvido, Eusebio Valero, A STABILITY ANALYSIS OF THE COMPRESSIBLE BOUNDARY LAYER FLOW OVER INDENTED SURFACES, Computers and Fluids (2017), doi: 10.1016/j.compfluid.2017.10.011

This is a PDF file of an unedited manuscript that has been accepted for publication. As a service to our customers we are providing this early version of the manuscript. The manuscript will undergo copyediting, typesetting, and review of the resulting proof before it is published in its final form. Please note that during the production process errors may be discovered which could affect the content, and all legal disclaimers that apply to the journal pertain. 


\section{Highlights}

- Stability of compressible boundary layer flow over indented surfaces is considered.

- Small surface indentations enhance certain flow instabilities.

- An increase in Mach number enhances further this behaviour.

- Amplification for deepest case are locally up to 20 times larger than in flat plate.

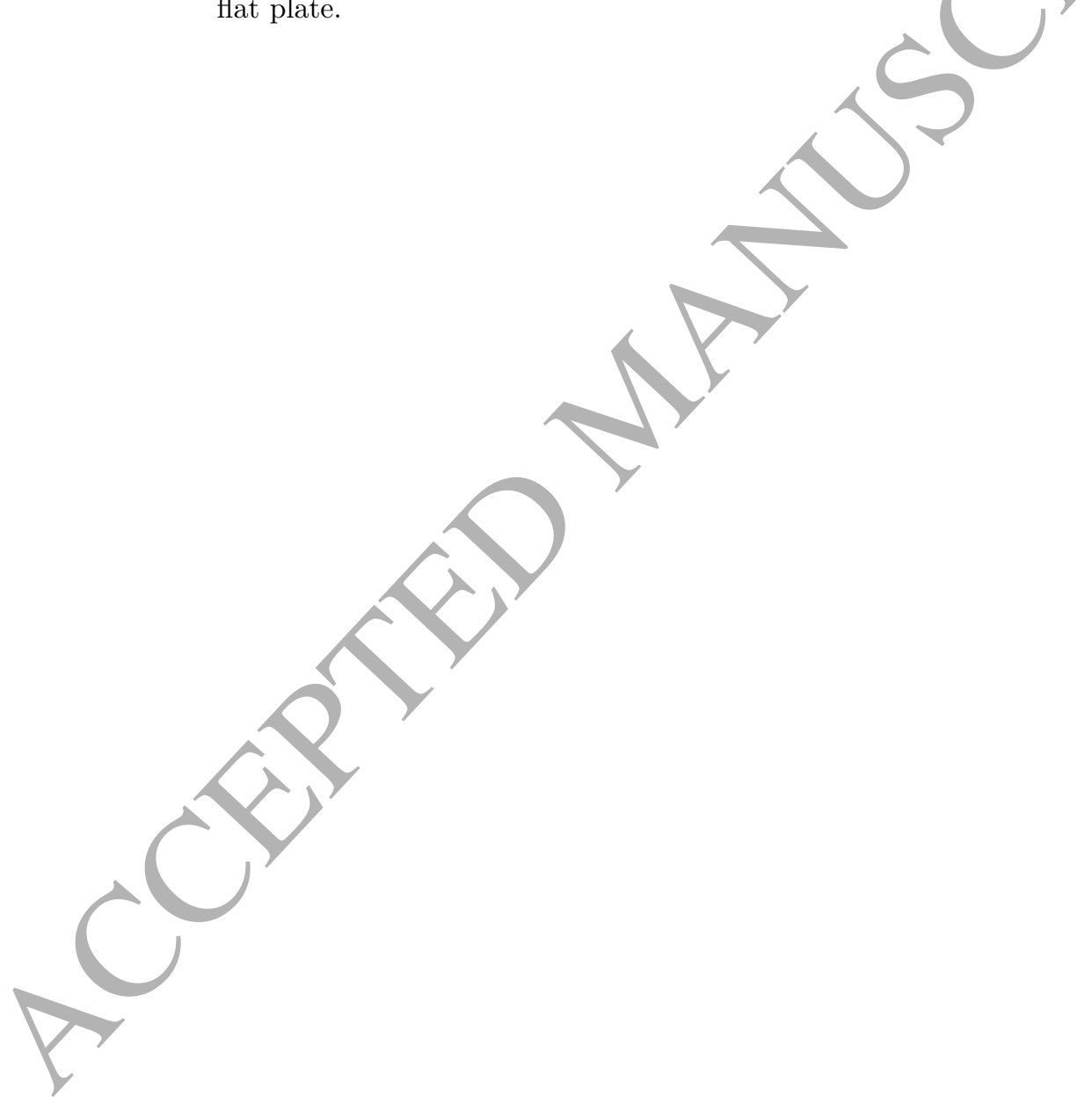


A STABILITY ANALYSIS OF THE COMPRESSIBLE BOUNDARY LAYER FLOW

OVER INDENTED SURFACES

Jesús Garicano-Mena ${ }^{\mathrm{a}, *}$, Esteban Ferrer ${ }^{\mathrm{a}}$, Silvia Sanvido $^{\mathrm{a}}$, Eusebio Valero $^{\mathrm{a}}$

${ }^{a}$ ETSIAE-UPM (School of Aeronautics) Plaza Cardenal Cisneros 3, E-28040 Madrid, Spain

Abstract

This contribution presents a stability analysis for compressible boundary layer flows over indented surfaces. Specifically, the effects of increasing depth $D / \delta^{*}$ and $M a_{\infty}$ number on perturbation time-decay ratés and spatial amplification factors are quantified and compared with those of an unindented configuration. The indented surfaces represent aeronautical lifting surfaces endowed with the smooth gap resulting when a filler material applied at the junction of leadingedge and wing-box components retracts upon its curing process. Since the configuration considered is such that the parallel/weakly-parallel assumptions are necessarily compromised, a global temporal stability analysis is considered in this study. Our analysis does not require a parallel flow constrain, and hence it is believed to be valid when two dimensional effects are relevant.

We find that small surface modifications enhance certain flow instabilities. An increase in $M a_{\infty}$ enhances further this behaviour: for the $D / \delta^{*}=1.5, M a_{\infty}=0.5$ case, amplification factors at a given location can be up to 20 times larger than those corresponding to the unindented case.

Keywords: stability analysis, compressible boundary layer flow, flat plate, indentation, high-order numerical method

2010 MSC: 76N20, 76E19, 76E09

\footnotetext{
${ }^{2}$ Fully documented templates are available in the elsarticle package on CTAN.

* Corresponding author

Email address: jesus.garicano.mena@upm.es (Jesús Garicano-Mena)
} 


\section{Introduction}

The aeronautics industry has shown an increased interest on natural laminar flow $(N L F)$ wings. These wings are carefully designed to maintain the flow under laminar conditions over a relatively large extent of the wing area. The

5 advantage is a lower skin friction and the consequent reduction in fuel consumption; this is achieved at the price of more stringent manufacturing tolerances.

From the manufacturing viewpoint, wings are assembled by joining several components, e.g. the main central wing box and the leading and trailing edges. The fitting between these elements is never perfectly tight: small grooves are always left at the wing-box/leading edge and trailing edge junctions. Filler materials, of resinous nature, are applied at these locations to alleviate the misfitting problem. However, since filler materials retract during its curing process, a small, possibly smooth indentation remains. The question arises then, whether this 15 smaller but somewhat unavoidable groove in the wing box/leading edge junction can enhance the growth of boundary layer instabilities. In such case, a significant forward movement of transition location would spoil the effort invested in the design of the natural laminar flow region.

20 It is well known that in real swept wings, transition to turbulence is mainly driven by cross-flow and Tollmien-Schlichting instability mechanisms [1]. However, and contrarily to more established wing concepts, $N L F$ wings operate at comparatively lower sweep angles [2]; this in turn translates in an increased relevance of Tollmien-Schlichting over cross-flow dominated transition mechanisms.

Spatial growth of Tollmien-Schlichting (or TS) structures is one of the avenues explaining laminar to turbulent transition. Through this mechanism, i.e. the natural transition scenario, the TS waves grow exponentially over a finite length, to then saturate and interact in a non-linear fashion, leading eventually to transo sition to turbulence. Alternatively, non-linear interactions may appear without 
a definite preliminary exponential growth phase [3] (hence the term bypass transition). These alternative mechanisms are not covered in this work.

In the aircraft industry, it is common practice to employ semi-empirical, but 35 extensively validated methods, to predict natural transition location for flows over wings and fuselages at flight conditions. The most common tools either perform a local stability analysis $[4,1]$ or solve the Parabolized Stability Equations [or $P S E, 5]$ upon a base state obtained numerically; application of the $e^{N}$ criteria $[6,7]$ allows then to predict approximate transition locations for natural scenarios, as long as the parallel or weakly parallel assumptions are fulfilled.

Whenever small surface imperfections are present, the preliminary exponential growth phase (natural transition) may be compromised, resulting possibly into a different transition location. In this case, the parallel hypothesis is not valid 45 anymore, and classical methods may have difficulties in predicting the modified transition location [2]. Alternative methods are needed to handle these situations.

Zahn \& Rist report in [8] a detailed analysis of the effect of deep gaps in laminar50 to-turbulent transition for a $M a_{\infty}=0.6$ flow, employing direct numerical simulation. They succeed in identifying an acoustic feedback mechanism between standing waves at the gap and the boundary layer, and derived a model that successfully accounts for amplification factor modifications. They also investigate the transition delay effect induced by a deep cavity placed before a forwardfacing step.

)

An alternative approach, based in the definition of a Local Scattering Problem, has been proposed recently in $[9,10,11]$. This method leads to an eigenvalue problem whose solution bridges the spatial behaviour much before and after the 60 scatter location (indentation, bump, different materials junction, ....) 
In this context, we propose to quantify the effect of small indentations by using global stability analysis techniques [12], since these do not rely on the parallel or weakly-parallel flow assumptions. Indeed, many contributions describe the 65 application of global techniques -both in its modal and non-modal variants- to study laminar separation bubbles on flat plate $(F P)$ configurations, be they generated by a convex bump [13, 14, 15], by a concave indentation [16] or by an adverse pressure gradient [17]. Alternatively, direct numerical simulation followed by solution of the linearised Navier-Stokes equations may be employed: 70 e.g. [18] investigates the effect of very small-scale, localised bumps and indentations on the Tollmien-Schlichting waves appearing on a FP configuration.

In this work, we aim at studying how the presence of an indentation modifies the stability characteristics of a canonical zero pressure gradient boundary 75 layer (or $B L$ ) over a flat plate. We specifically seek to quantify the effects of increasing indentation depth and flow compressibility (i.e. Mach number) on the linear stability (i.e. the spectrum and amplification factors) by means of global stability tools. In line with most of the studies mentioned above, the flow is considered bidimensional

80

The rest of the document is structured as follows: next section describes the flow configurations considered and presents the tool chain employed in our study. Section 3 gathers the results and discussions. Finally, section 4 summarises our conclusions.

85

\section{Flow configuration and numerical methods}

We study a zero pressure gradient boundary layer flow over a flat plate geometry that includes a smooth groove or indentation. The indentation, of infinite spanwise extent, sits at a certain distance downstream of its leading edge (see 90 section 2.1 for the problem description). We proceed -as in a classical stability analysis- by obtaining first a steady (numerical) solution to the flow governing 
equations: the base flow ( $c f$. sections 2.2 and 2.3); a linear perturbation of this basic flow solution and its subsequent expansion in terms of Fourier modes allows then to assemble a discrete eigenvalue problem, (or EVP, cf. section 2.4).

${ }_{95}$ The spectral information (eigenvalues and eigenfunctions) retrieved is analysed along two dimensions: on the one hand, the eigenvalue locations in the complex plane; on the other hand, the spatial evolution of individual components along the streamwise direction, as given by their amplification factors (section 2.5).

\subsection{Problem description}

We consider compressible boundary layer flows with $\operatorname{Re}_{\delta^{*}(x)} \in[610,1050]$ at upstream Mach numbers $M a_{\infty}=0.1$ and 0.5. The incompressible boundary layer flow over a flat plate configuration is, in the range of $R e_{\delta^{*}(x)}$ considered, convectively unstable [4], and has been addressed in $[19,20]$.

The Reynolds number is based on a displacement thickness $\delta^{*}(x)$ :

$$
R e_{\delta^{*}}=\frac{\rho_{\infty} U_{\infty} \delta^{*}(x)}{\mu_{\infty}},
$$

where $\rho_{\infty}, U_{\infty}$ and $\mu_{\infty}$ are the density, speed and dynamic viscosity upstream.

Figure 1 shows the computational domain studied: it is rectangular in shape, of length $L_{x}$ and height $L_{z}$, and the air flows from left to right. The leading edge of the flat plate is not simulated, instead a solution to the compressible boundary layer equations at the corresponding $M a_{\infty}$ is imposed at the leftmost edge of the computational domain, i.e. the inlet. The choice of the domain extent is partly guided by previous results on incompressible $B L$ flows at the same $R e_{\delta^{*}}[19,20]$. Specifically, $L_{z} / \delta^{*}$ needs to be chosen large enough so neither the $B L$ growth nor the global eigenfunctions are artificially constrained, see [12]; this consideration becomes more and more restrictive as both $M a_{\infty}$ and $D / \delta^{*}$ increase.

The isolated indentation, when present, is located at a distance $x_{c}$ from the left edge and is characterised by its breadth $L$ and depth $D$. The notch considered 


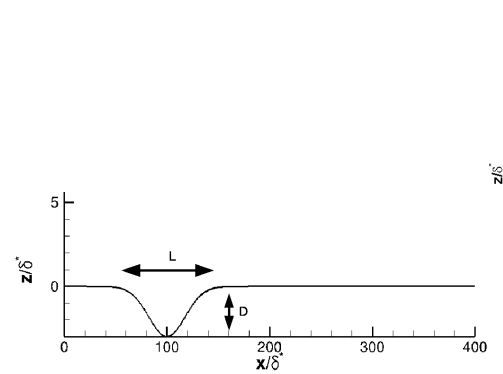

(a)

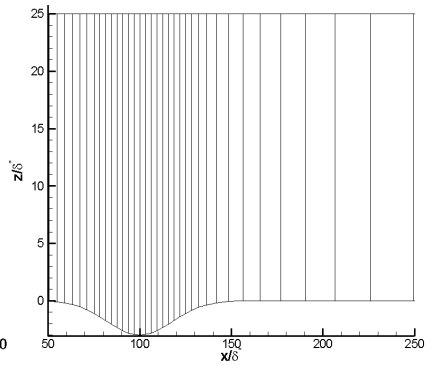

(b)

Figure 1: Computational domain definition: wall profile (a); and part of the Spectral Element mesh employed (b, every other element shown) for $D / \delta^{*}=1.5$. Axes are not to scale.

\begin{tabular}{cc||ccc||c|c|c}
$R e_{\delta^{*}}$ & $M a_{\infty}$ & $L_{x} / \delta^{*}$ & $L_{z} / \delta^{*}$ & $x_{c} / \delta^{*}$ & $L / \delta^{*}$ & $D / \delta^{*}$ & $L / D$ \\
\hline 610 & 0.1 and 0.5 & 400 & 40 & 100 & 50 & $0,1,1.5$ & $\infty, 50,33.3$
\end{tabular}

Table 1: Configurations considered.

presents a smooth, Gaussian-like profile given as $z=-D \exp \left(\frac{x-x_{c}}{L / 2}\right)^{2}$.

All the geometrical parameters defining the problem are non-dimensionalised with the mass displacement thickness $\delta^{*}$ at the leftmost edge of the domain. In this study we fix the groove extent $L / \delta^{*}$ and location $x_{c} / \delta^{*}$ and vary the groove depth $D / \delta^{*}$ and the upstream $M a_{\infty}$ number [21]; table 1 summarises the different configurations considered. Notice that the range for the ratio $L / D$ here included is essentially different from previous studies on rectangular low aspect ratio cavities - $L / D \in(1 / 5,4)$ - at high Reynolds and Mach numbers described e.g. in $[22,23,8]$.

\section{Governing equations}

We are interested in flows governed by the compressible Navier-Stokes equations, that once expressed in terms of non-dimensional, conserved variables $\mathbf{U}=\left[\rho, \rho \vec{v}^{t}, \rho E\right]^{t}$ (mass, momentum and total energy per unit volume), can 
be written in compact vector form as:

$$
\frac{\partial \mathbf{U}}{\partial t}+\mathcal{N}(\mathbf{U})=\overrightarrow{0}
$$

where $\mathcal{N}$ is the divergence of the flux tensor:

$$
\mathcal{N}=\nabla \cdot \overline{\bar{F}}(\mathbf{U})
$$

and $\overline{\bar{F}}$ gathers convective and diffusive effects:

$$
\overline{\bar{F}}(\mathbf{U})=\left(\begin{array}{c}
\rho \vec{v} \\
\rho \vec{v} \cdot \vec{v}^{t}+p \overline{\bar{I}}-\overline{\bar{T}} \\
\rho H \vec{v}-\overline{\bar{T}} \cdot \vec{v}+\vec{q}
\end{array}\right)
$$

In equation (2) above, $p$ and $\rho H$ stand for thermodynamic pressure and total enthalpy per unit volume, respectively; $\overline{\bar{I}}$ is the identity matrix.

A Newtonian behaviour is assumed, the viscous stress tensor $\overline{\bar{T}}$ is therefore given 140 by the expression:

$$
T_{i, j}=\frac{1}{R e}\left[\frac{\partial u_{i}}{\partial x_{j}}+\frac{\partial u_{j}}{\partial x_{i}}-\frac{2}{3} \frac{\partial u_{k}}{\partial x_{k}} \delta_{i, j}\right],
$$

while the heat flux vector $\vec{q}$ is given by Fourier law:

$$
q_{i}=-\frac{1}{\operatorname{Re} \operatorname{Pr}} \frac{\partial T}{\partial x_{i}}
$$

\subsection{Base flow computation}

In order to solve system (2), we employ a high-order, staggered multi-domain Spectral Element Method $(S E M)$ technique, described in detail in [24, 25]. The numerical tool solves the strong form of the compressible Navier-Stokes equations using collocated values for $\mathbf{U}$ at Gauss-Chebychev nodes; the flux tensor $\overline{\bar{F}}$ is discretised at Gauss-Chebychev-Lobatto nodes to ensure inter-element continuity. The solution and the flux tensor are expressed as:

$$
\mathbf{U}^{h}=\sum_{e=1}^{N_{D o m}} \sum_{i, k=1}^{p_{x}+1, p_{z}+1} \mathbf{U}_{i, k}^{e} \Phi_{i, k}^{e}(x, z), \quad \overline{\bar{F}}^{h}=\sum_{e=1}^{N_{D o m}} \sum_{i, k=1}^{p_{x}+1, p_{z}+1} \overline{\bar{F}}_{i, k}^{e} \Psi_{i, k}^{e}(x, z),
$$


where $\Phi_{i, k}^{e}(x, z)$ and $\Psi_{i, k}^{e}(x, z)$ are the basis functions obtained as a tensor product of one-dimensional Legendre polynomials (orders $p_{x}$ and $p_{z}$ ). Note that the flux tensor discretisation employs a higher-order polynomial, so that its divergence has the same order as the one used for the solution.

The spatially discretised time varying and non-dimensional form of system (2) is:

$$
\mathcal{M} \frac{\partial \mathbf{U}^{h}}{\partial t}=\mathcal{N}\left(\mathbf{U}^{h}\right)
$$

The non-linear discrete operator $\mathcal{N}\left(\mathbf{U}^{h}\right)$ above gathers the convective and diffusive contributions, while $\mathcal{M}$ is the mass matrix resulting from the $S E M$ spatial discretisation. It is convenient to proceed by redefining

$$
\frac{\partial \mathbf{U}^{h}}{\partial t}=\mathbf{G}\left(\mathbf{U}^{h}\right) ; \text { where } \mathbf{G}\left(\mathbf{U}^{h}\right)=\mathcal{M}^{-1} \mathcal{N}\left(\mathbf{U}^{h}\right) .
$$

155

We use a marching procedure in pseudo-time [26] to converge system (9) towards a steady state solution, reached whenever the pointwise maximum of $\frac{\partial \mathbf{U}}{\partial t}$ term falls below $1 \times 10^{-9}$. This methodology enables stability studies upon steady base flows and instabilities of convective nature. Additional details on the base

At the interfaces between adjacent domains, the advective contributions are computed with the Roe approximated Riemann solver, while viscous terms are simply averaged. High-order representation of curved boundaries is achieved by means of a conformal mapping between the physical and computational domains: this succeeds at representing accurately the wall surface, while avoiding spurious perturbations in the solution.

At the left, top and right boundaries the compressible $B L$ profile at the corresponding $R e_{\delta^{*}(x)}$ is imposed through a Riemann flux solver. Additionally, the viscous fluxes are extrapolated from the interior domain. For the wall, an 
adiabatic, no-slip boundary condition is enforced.

\subsection{Eigenvalue problem formulation and stability analysis}

Let $\mathbf{U}_{b}^{h}$ be a (discrete) steady solution to system (9), obtained with the $S E M$ solver described above ${ }^{1}$. Consider next a linearly perturbed base flow, say:

$$
\mathbf{U}_{*}=\mathbf{U}_{b}^{h}+\mathbf{U}^{\prime} \text {, with }\left\|\mathbf{U}^{\prime}\right\| \ll\left\|\mathbf{U}_{b}^{h}\right\| \text {. }
$$

Aiming at a Global (modal) temporal linear stability analysis [12], the perturbation $\mathbf{U}^{\prime}$ is expanded as:

$$
\mathbf{U}^{\prime}=\hat{\mathbf{U}}(x, z) e^{-i \omega t}+\text { c.c. },
$$

where $\omega \in \mathbb{C}$ and c.c. stands for complex conjugate. Substitution of equation (11) into (9) and subsequent linearisation (i.e. using a first order Taylor series approximation around the base flow) lead to a (discrete) linearised system of equations which, when rearranged as the block-vector $\mathcal{U}$, can be written as the eigenvalue problem:

$$
\mathcal{A U}=-i \omega \mathcal{U}
$$

Matrix $\mathcal{A}$ is the Jacobian $\frac{\partial \mathbf{G}^{h}}{\partial \mathbf{U}_{b}^{h}}$. In this work, the Jacobian matrix $\mathcal{A}$ has been computed numerically using a complex-step approximation [27], which has already been used for stability analysis by the authors in [28].

The complex-step derivative approximation is accomplished by approximating a nonlinear function $g$ (i.e. any of the components of $\mathbf{G}$ ) with a complex variable using a Taylor series expansion:

$$
g(x+i \epsilon)=g(x)+\frac{d g}{d x} i \epsilon-\frac{d^{2} g}{d x^{2}} \frac{\epsilon^{2}}{2}-\ldots
$$

\footnotetext{
${ }^{1}$ In this work, the stability analysis is performed on the same domain where the base
} flow has been computed: contrarily to other approaches $[19,16]$, no interpolation is applied between base flow and stability domains. 
taking the imaginary part and neglecting terms of order $\epsilon^{2}$ and higher:

$$
\frac{d g}{d x}=\frac{\operatorname{Im}[g(x+i \epsilon)]}{\epsilon} .
$$

The advantages of the complex-step approximation approach over a standard finite differencing include: 1) it provides second-order accuracy with a single function evaluation , 2) the Jacobian approximation is not subject to subtractive cancellations inherent in roundoff errors, and 3) it is easy to implement in a black-box manner, thereby making applicable to general nonlinear functions. On the downside part, this approach requires to implement a complex version of the numerical fluxes $\overline{\bar{F}}^{h}$.

195

Assembling the eigenvalue problem in equation (12) through the discrete approach $[29,28]$ implies that the Jacobian matrix $\mathcal{A}$ is built by repeated application of the base flow solver. In this sense, boundary conditions $(B C s)$ for the eigenfunctions $\mathcal{U}$ are defined by the base flow solver and the complex-step derivative approximation just described. Namely, BCs for the EVP are inherited from the base flow solver. Thus, in view of how $B C s$ are enforced in the base flow problem, $B C s$ for the eigenfunctions $\mathcal{U}$ are equivalent to Robin conditions.

205 A Shift and Invert methodology [30], relying on an Arnoldi iteration technique [31] implemented by means of MUMPS library [32], allows to obtain approximations to the spectrum of $\mathcal{A}$. The number of eigenvalues/eigenvectors retrieved is precisely the dimension of the Krylov space employed, $N_{K}$.

210 Complex eigenvalues $\omega=\omega_{R}+i \omega_{I}$ obtained from equation (12) characterise the corresponding eigenfunctions $\mathcal{U}$ : the real part $\omega_{R}$ is the mode angular pulsation (related to its temporal frequency) while the imaginary part $\omega_{I}$ establishes how fast the mode grows/decays exponentially in time. 


\subsection{Amplification factors}

In order to quantify in a consistent manner the effect of the indentation on the spatial growth of the TS-like eigenmodes, we follow [19] and define the spatial amplification factor of the eigenfunctions as:

$$
A(x)=\sqrt{\int_{z_{\min }(x)}^{L_{z}}\left(\delta u^{\dagger} \delta u+\delta w^{\dagger} \delta w\right) d z},
$$

where $\delta u$ and $\delta w$ are the velocity components of the 2D global perturbation;

superscript $\dagger$ indicates complex conjugation.

Since the eigenvalue problem in equation (12) is formulated in terms of the conserved variables, $\delta u$ and $\delta w$ are not directly available; but can be retrieved straightforwardly as:

$$
\delta u=\frac{\delta(\rho u)-u_{b} \delta \rho}{\rho_{b}}, \delta w=\frac{\delta(\rho w)-w_{b} \delta \rho}{\rho_{b}},
$$

where $u_{b}=\rho u_{b} / \rho_{b}$ and $w_{b}=\rho w_{b} / \rho_{b}$ are computed from the base flow $\vec{U}_{b}$.

\section{Results and discussion}

\subsection{Base flows}

Solutions for the unindented flat plate and two different depths for different upstream $M a_{\infty}$ numbers (see Table 1 ) have been computed. In every case, the domain has been divided in $N_{D o m}=48$ spectral elements distributed along the streamwise direction, with refinement near the indentation region (figure $1 \mathrm{~b}$ ). Different intra-element resolutions $p_{x} \times p_{z}$ have been considered; $p_{x}$ and $p_{z}$ are the polynomial orders of the underlying basis functions along the $x$ and $z$ directions respectively. For all the configurations discussed in this work, the residuals decreased by at least nine orders of magnitude.

In figure 2, we address the accuracy and the sensitivity of the numerical tool employed. Figure 2a compares the skin friction coefficient $C_{f}$ for the unindented low $M a_{\infty}=0.1$ case (intra-element resolution $p_{x} \times p_{z}=10 \times 60$ ) against 
that corresponding to a incompressible Blasius profile: these curves overlap. Figure $2 \mathrm{~b}$ presents the sensitivity of the $C_{f}$ coefficient to intra-element grid resolution for the most challenging $D / \delta^{*}=1.5, M a_{\infty}=0.5$ case: there is barely any appreciable difference for the different resolutions considered. For reasons explained in section 3.2 , we retain results computed with the $p_{x} \times p_{z}=10 \times 60$ resolution.

The effect of the notch is to deflect the incoming $B L$ flow; of course, the deeper the indentation the larger the flow deflection. For the case $D / \delta^{*}=1$ the flow remains attached, as revealed by skin friction $D 1$ in figures $3 \mathrm{c}-3 \mathrm{~d}$; for the deepest case the flow separates from the wall and a steady-state recirculation zone, confined in the groove, is established (see figures $3 a-3 b$ ), thus invalidating the parallel flow assumption. This fact is confirmed by curves $D 1.5$ in figures $3 \mathrm{c}-3 \mathrm{~d}$.

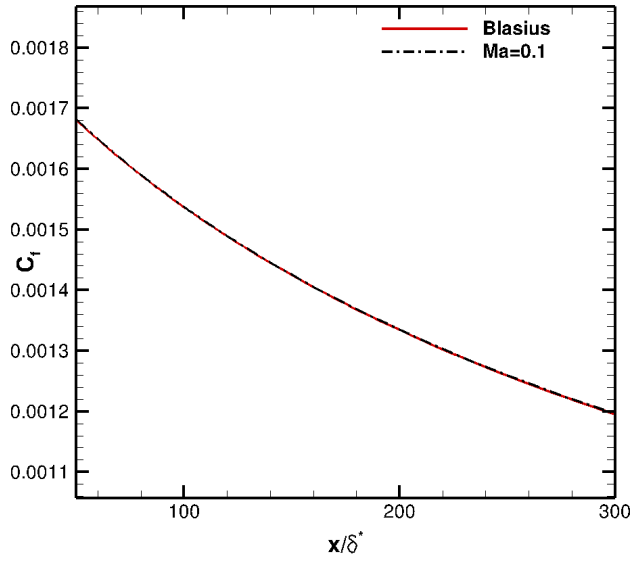

(a)

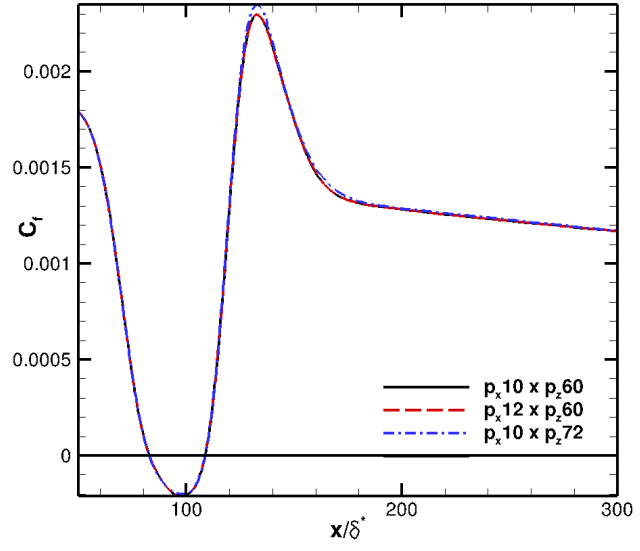

(b)

Figure 2: (a) Skin friction for the $M a_{\infty}=0.1 F P\left(p_{x} \times p_{z}=10 \times 60\right)$ case against that corresponding to a Blasius profile (curves overlap); (b) Effect of numerical resolution on skin friction for $M a_{\infty}=0.5, D / \delta^{*}=1.5$. 


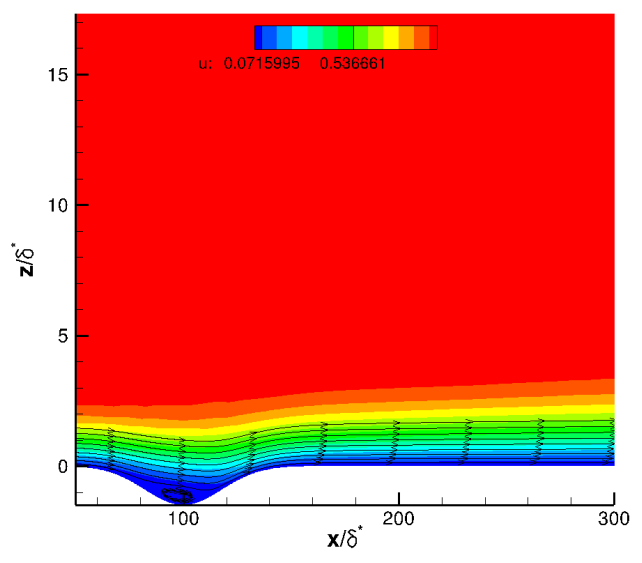

(a) $M a_{\infty}=0.1$

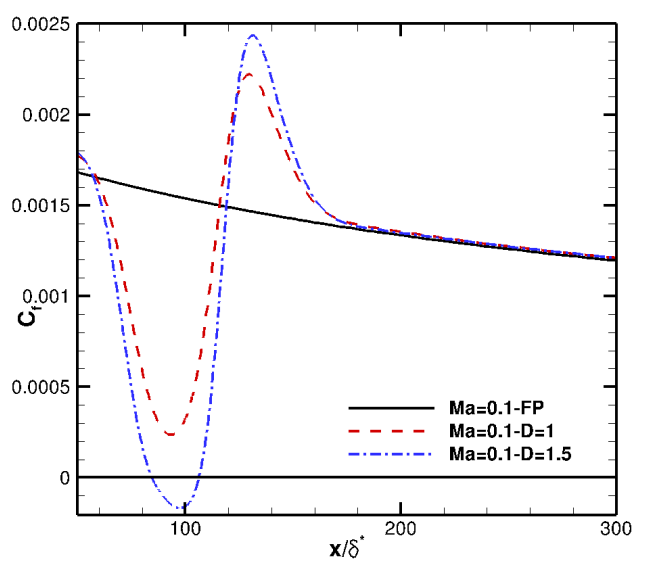

(c) $M a_{\infty}=0.1$

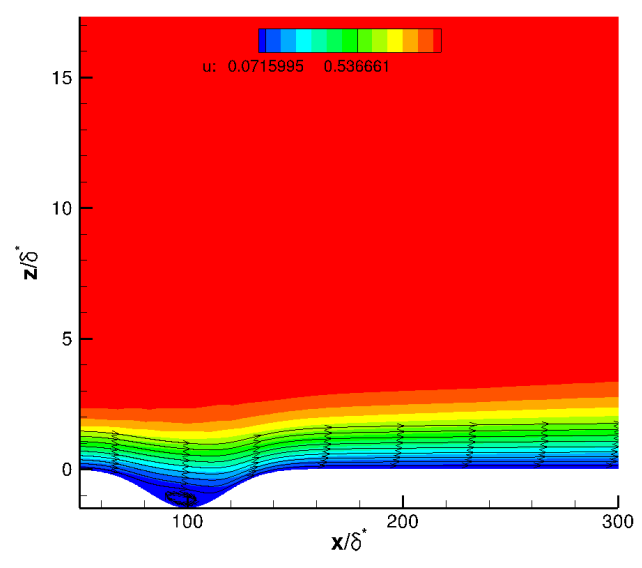

(b) $M a_{\infty}=0.5$

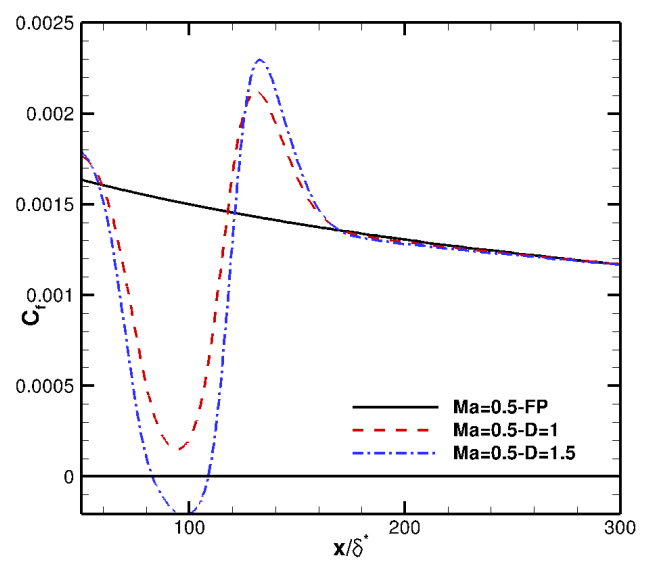

(d) $M a_{\infty}=0.5$

Figure 3: Horizontal velocity component for $D / \delta^{*}=1.5$ case with $M a_{\infty}=0.1$ (a) and $M a_{\infty}=$ 0.5 (b) conditions. Skin friction for FP (-), and depths $D / \delta^{*} 1(---)$ and $1.5(-.--)$ for both $M a_{\infty}=0.1$ (c) and $M a_{\infty}=0.5$ (d). 


\subsection{Temporal modes}

Figures $4 \mathrm{a}-4 \mathrm{~b}$ show the temporal spectra for the different configurations de-

Concerning the accuracy of the eigenmodes retrieved, all of them fulfil that $\|\mathcal{A U}+i \omega \mathcal{U}\|_{\infty} \leq 1 \times 10^{-11}$ with $\|\mathcal{U}\|_{2}=1$. In both cases a Krylov space dimension of $N_{K}=1000$ has been considered, as increasing $N_{K}$ to 1500 leads to no change in the spectra over the region of interest. 


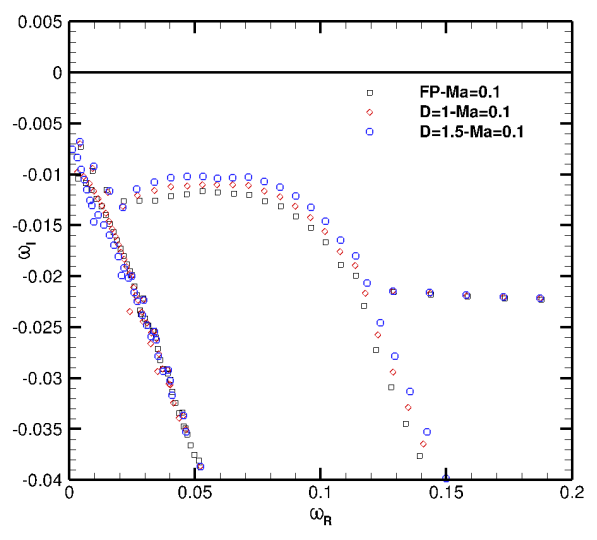

(a) $M a_{\infty}=0.1$

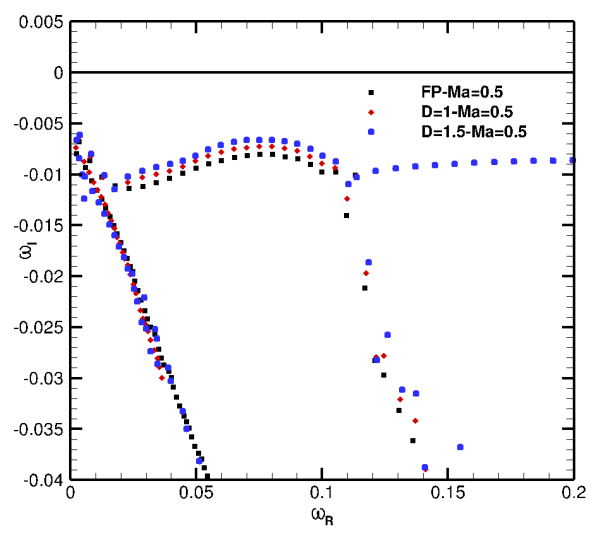

(b) $M a_{\infty}=0.5$

Figure 4: Indentation depth effect on global temporal spectra for both upstream Mach numbers $M a_{\infty}=0.1$ (a) and 0.5 (b).

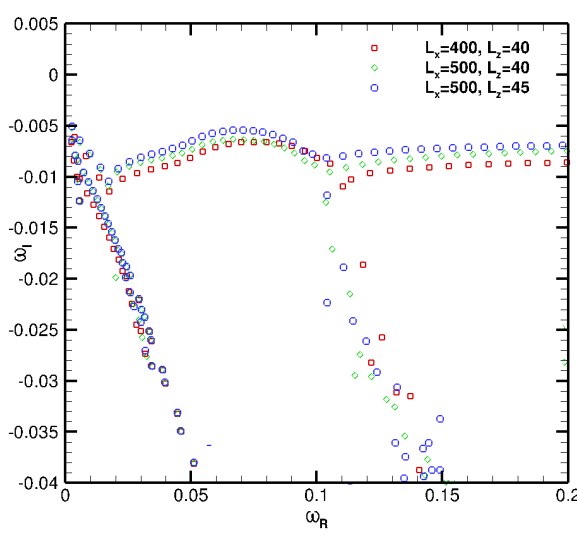

(a) Domain size.

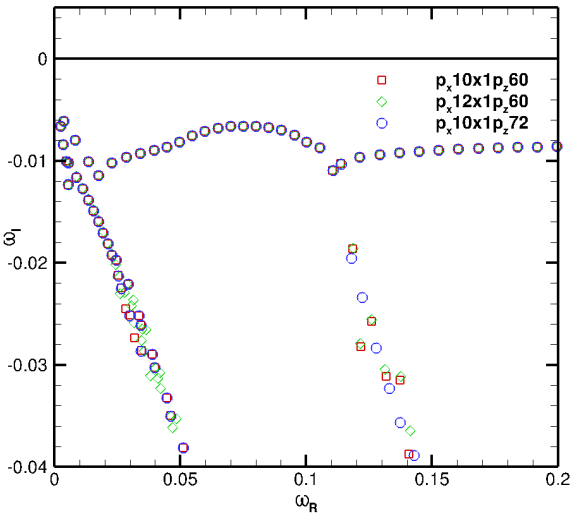

(b) Polynomial order.

Figure 5: Grid study for $M a_{\infty}=0.5, D / \delta^{*}=1.5$ case: sensitivity to boundary size location (a) and polynomial order (b). 


\begin{tabular}{|c|c|c|c|c|c|c|c|c|}
\hline & & \multicolumn{3}{|c|}{$F P$} & \multicolumn{3}{|c|}{$D / \delta^{*}=1.5$} & \multirow{2}{*}{$\begin{array}{l}\text { Change with } D / \delta^{*} \\
\frac{\Delta^{A} /\left.A_{0}\right|_{x / \delta^{*}}=120}{A /\left.A_{0}\right|_{x / \delta^{*}}=120} \\
\end{array}$} \\
\hline & & $\omega_{R}$ & $\omega_{I}$ & ${ }^{A} /\left.A_{0}\right|_{x / \delta^{*}}=120$ & $\omega_{R}$ & $\omega_{I}$ & ${ }^{A} /\left.A_{0}\right|_{x / \delta^{*}}=120$ & \\
\hline \multirow{2}{*}{$M a_{\infty}$} & 0.1 & 0.0529 & -0.0117 & 1.86 & 0.0530 & -0.0102 & 3.58 & 0.924 \\
\hline & 0.5 & 0.0837 & -0.0081 & 1.75 & 0.0802 & -0.0067 & 6.60 & 2.77 \\
\hline \multirow[b]{2}{*}{ Change with $M a_{\infty}$} & & & & -0.059 & & & 0.84 & \\
\hline & & & & $\frac{\Delta A /\left.A_{0}\right|_{x / \delta^{*}=120}}{A /\left.A_{0}\right|_{x / \delta^{*}}=120}$ & & & $\frac{\Delta A /\left.A_{0}\right|_{x_{1 / \delta^{*}}=120}}{A /\left.A_{0}\right|_{x / \delta^{*}=120}}$ & \\
\hline
\end{tabular}

Table 2: Angular pulsation $\omega_{R}$, temporal amplification $\omega_{I}$ and spatial amplification $A / A_{0}$ at $x / \delta^{*}=120$, and their relative changes with $D / \delta^{*}$ and $M a_{\infty}$ for eigenfunction $\mathrm{M} 1$.

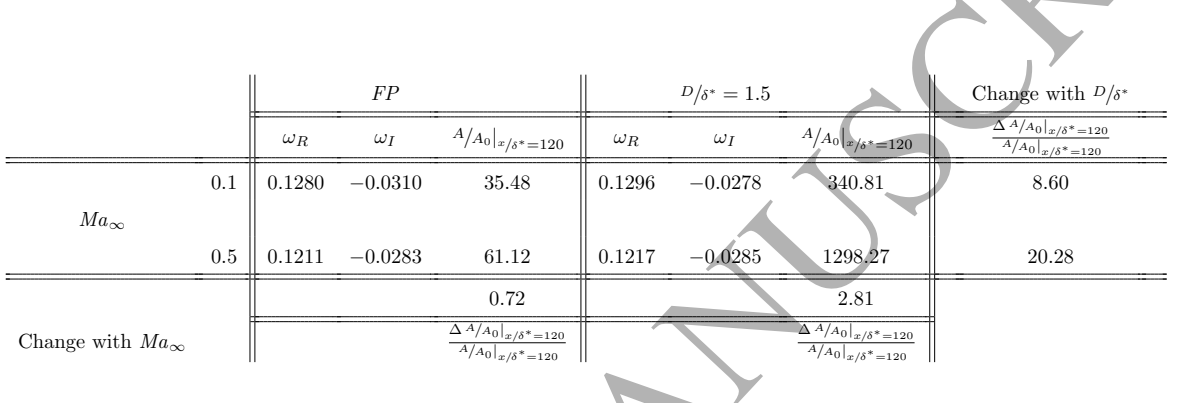

Table 3: Angular pulsation $\omega_{R}$, temporal amplification $\omega_{I}$ and spatial amplification $A / A_{0}$ at $x / \delta^{*}=120$, and their relative changes with $D / \delta^{*}$ and $M a_{\infty}$ for eigenfunction M2.

\begin{tabular}{|c|c|c|c|c|c|c|c|}
\hline & & \multicolumn{5}{|c|}{$D / \delta^{*}=1.5$} & \multirow{2}{*}{$\begin{array}{c}\text { Change with } D / \delta \\
\frac{\Delta A /\left.A_{0}\right|_{x / \delta^{*}=120}}{A /\left.A_{0}\right|_{x / \delta^{*}=120}}\end{array}$} \\
\hline & & $\omega_{R}$ & $A /\left.A_{0}\right|_{x / \delta^{*}=120}$ & $\omega_{R}$ & $\omega_{I}$ & $A /\left.A_{0}\right|_{x / \delta^{*}=120}$ & \\
\hline & 0.1 & & 3.41 & 0.1288 & -0.0215 & 59.84 & 16.55 \\
\hline & 0. & 0.1 & 1.33 & 0.1292 & -0.094 & 8.91 & 5.70 \\
\hline & & & -0.61 & & & -0.85 & \\
\hline & & & $\frac{\Delta A /\left.A_{0}\right|_{x / \delta^{*}=120}}{A /\left.A_{0}\right|_{x / \delta^{*}=120}}$ & & & $\frac{\left.\overline{\Delta A / A_{0}}\right|_{x / \delta^{*}=120}}{A /\left.A_{0}\right|_{x / \delta^{*}=120}}$ & \\
\hline
\end{tabular}

Table 4: Angular pulsation $\omega_{R}$, temporal amplification $\omega_{I}$ and spatial amplification $A / A_{0}$ at $x / \delta^{*}=120$, and their relative changes with $D / \delta^{*}$ and $M a_{\infty}$ for eigenfunction M3.

At the sight of figures $4 \mathrm{a}-4 \mathrm{~b}$ (see also figure 6 ), we conclude that the Orr-like branch is practically insensitive to increasing both depth $D / \delta^{*}$ and $M a_{\infty}$. Since our objective is to study TS based transition, we focus on TS-like structures.

${ }_{285}$ As shown in figures 4a-4b, the effect of the progressive increase of groove depth $D / \delta^{*}$ is, irrespective of $M a_{\infty}$, to reduce the temporal decay rate $\omega_{I}$ for those 
modes with angular pulsation $\omega_{R} \in[0.02,0.12]$. This increase in $\omega_{I}$ does not destabilises the modes, however. Observe also how eigenvalues with pulsation lower than 0.02 and higher than 0.12 over the quasi-horizontal branch piece are insensitive to $D / \delta^{*}$ when $M a_{\infty}$ is fixed.

The effect of $M a_{\infty}$ is evident in figures $6 \mathrm{a}, 6 \mathrm{~b}$ and $6 \mathrm{c}$. At each depth considered, eigenvalues for the high $M a_{\infty}=0.5$ case are systematically less temporally stable (i.e. $\omega_{I}$ are comparatively larger). The effect of $M a_{\infty}$ is specially signification for the higher $\omega_{R}$ end of the TS branch, where $M a_{\infty}=0.5$ modes are much less temporally stable than their $M a_{\infty}=0.1$ counterparts.

For the remainder of the discussion we will focus on three eigenpairs, shown in figure $6 \mathrm{c}$ corresponding to $M a_{\infty}=0.5$ and $D / \delta^{*}:$ M1 are the modes with largest temporal amplification; the choice of M2 is justified in section 3.3; M3 is chosen as representative of the modes in the large pulsation range. Tables 2-4 gather additional information on these modes for the $F P$ and the $D / \delta^{*}=1.5$ cases.

Let us describe now the shape of modes M1, M2 and M3 highlighted in figure 6c.

305 Figure 7a shows M1: it presents the characteristic TS structure, with a relatively long wavelength. Higher frequency modes M2 and M3 display also a TS character, with progressively shorter wavelengths than M1, figures $7 \mathrm{~b}$ and $7 \mathrm{c}$. Notice also how M2 and M3 differ specially in the groove region, as M3 is (visually) appreciable already before the leading edge of the indentation and its magnitude is specially intense in the trailing edge of the indentation, near the reattachment region of the flow. And yet another difference, M1 and M2 grow monotonically over the region of interest while M3 decays after an initial growth behaviour (see also figure 8). 


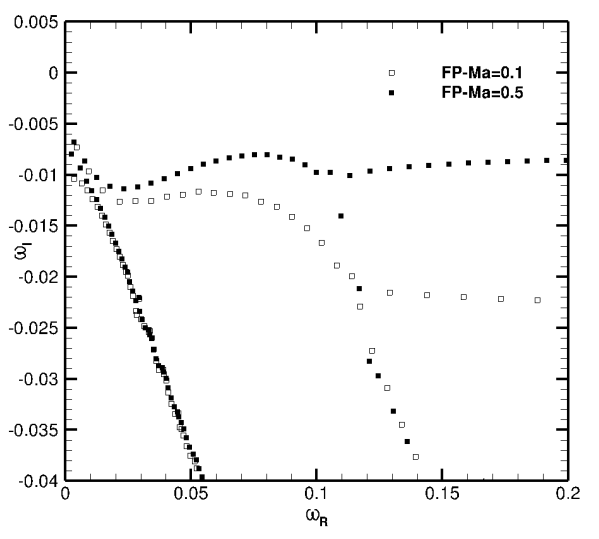

(a) FP

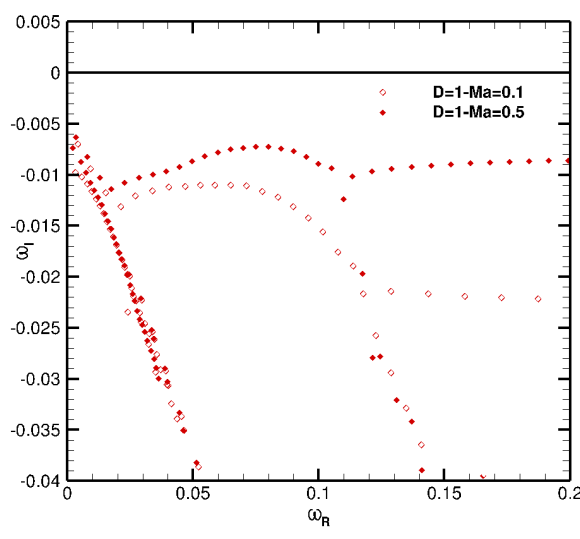

(b) $D / \delta^{*}=1$

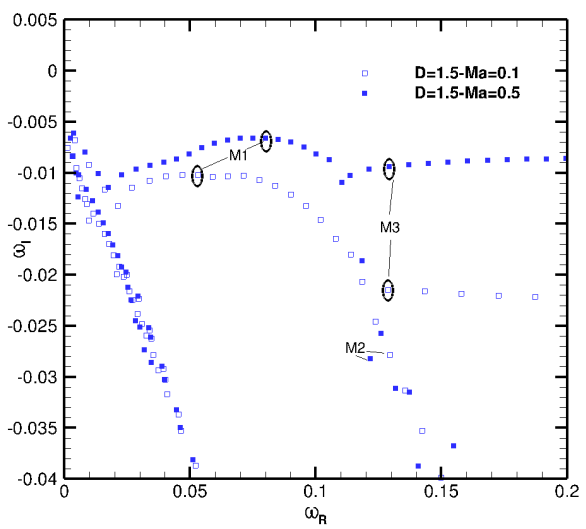

(c) $D / \delta^{*}=1.5$

Figure 6: Influence of $M a_{\infty}$ on spectra for $F P(\mathrm{a}), D / \delta^{*}=1$ (b) and $D / \delta^{*}=1.5$ (c). 


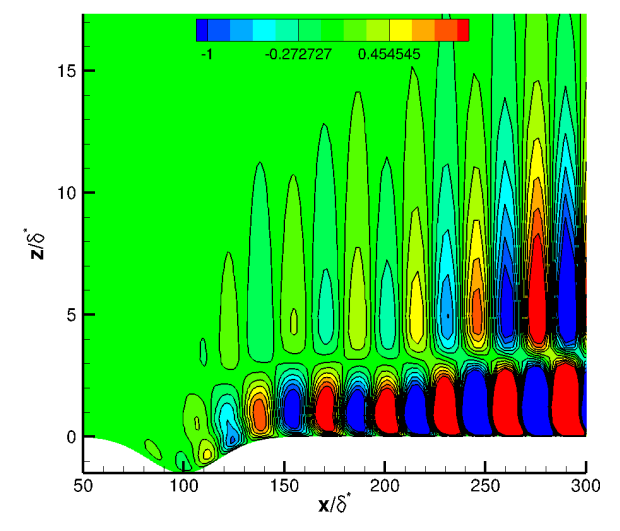

(a) M1 for $M a_{\infty}=0.5$ and $D / \delta^{*}=1.5$

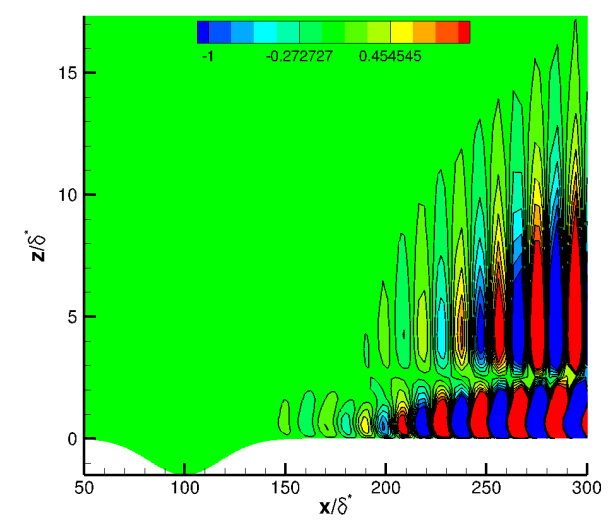

(b) M2 for $M a_{\infty}=0.5$ and $D / \delta^{*}=1.5$

1
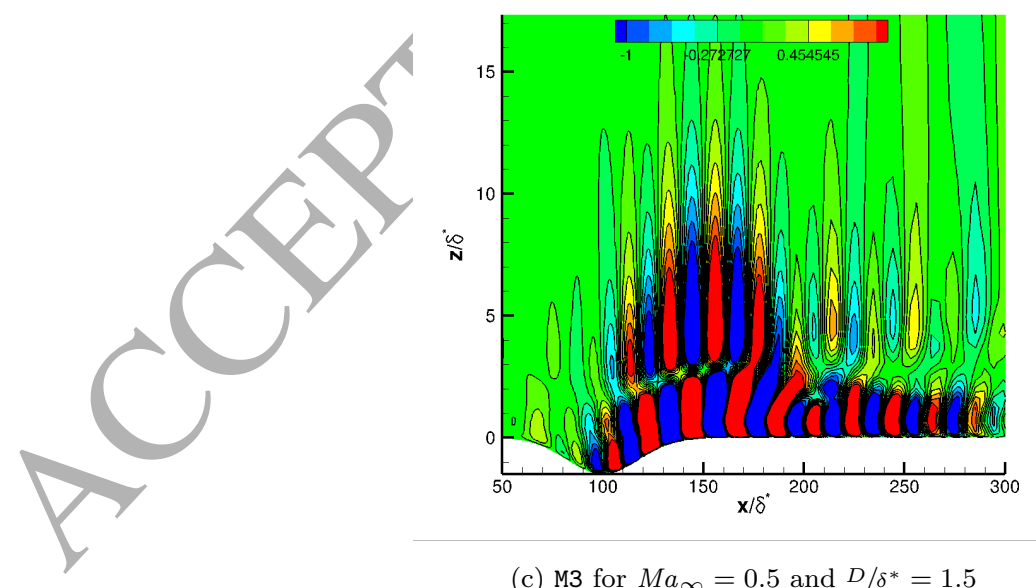

(c) M3 for $M a_{\infty}=0.5$ and $D / \delta^{*}=1.5$

Figure 7: Real part of the $x$-momentum perturbation for eigenfunctions M1 (a), M2 (b), and M3 (c) for $D / \delta^{*}=1.5, M a_{\infty}=0.5$. 


\subsection{Amplification factors}

In this section we analyse the amplification factors -equation (15)- to quantify the spatial growth of the different eigenmodes considered. Amplification factors are non-dimensionalised with their value $A_{0}=A\left(x / \delta^{*}=50\right)$, well before the indentation: subsequent figures actually display the ratio $A(x) / A_{0}$ over the range $x / \delta^{*} \in[50,300]$.

In order to measure the relative growth of the spatial modes, we have recourse to the $e^{N}$ semi-empirical criteria. Engineering practice assumes laminar to turbulent transition $[6,7]$ whenever a linear perturbation has grown $e^{8}$ to $e^{10}$ times.

Figure 8 shows amplification factors for the deepest case $D / \delta^{*}=1.5$ at both Mach numbers for modes M1, M2 and M3 in previous section. Eigenfunctions with pulsation $\omega_{R} \approx 0.121$ for $M a_{\infty}=0.5\left(\omega_{R} \approx 0.130\right.$ for $\left.M a_{\infty}=0.1\right)$ are those with the fastest and largest spatial growth, for both the $M a_{\infty}$ considered. This is the reason supporting the choice of M2 in figure $6 \mathrm{c}$.

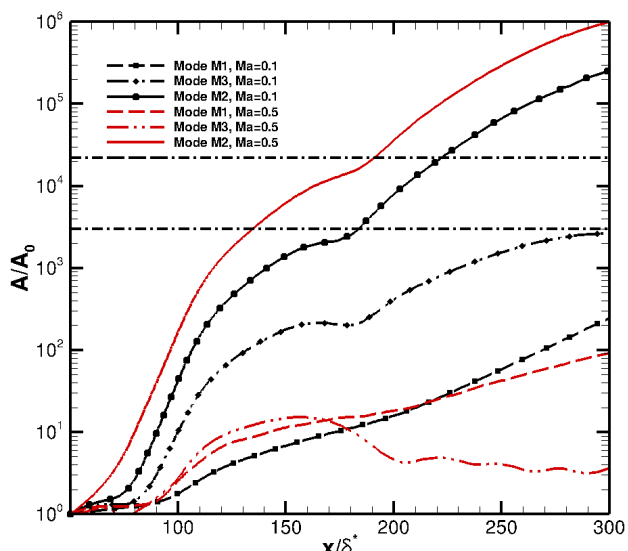

Figure 8: Amplification factors, for $D / \delta^{*}=1.5$ configuration at $M a_{\infty}=0.1,0.5$. Most unstable (M1), most spatially amplified (M2) and high frequency (M3) modes considered. 
Observe how the associated amplification factors for both $M a_{\infty}$ numbers in the FP case do not differ significantly, figure $9 \mathrm{a}$ and table 3 . However, as depth is increased, an increased $M a_{\infty}$ results in faster spatial growth, figures $9 \mathrm{~b}-9 \mathrm{c}$.

Figure 10 brings another perspective of M2 behaviour, complemented with M1 and M3 sensititivities to $D / \delta^{*}$ and $M a_{\infty}$.

Modes M1 and M2 -both in the pulsation range where spectra is sensitive to ${ }^{D} / \delta^{*}$ share a similar behaviour (figures 10a-10d), where the streamline deflection induced by the indentation alters the slope of ratio $A(x) / A_{0}$; this modification increases with depth (and base flow separation), resulting in an increase of up to hundred times over the extent of the indentation width. By the end of the indentation, the slope of $A(x) / A_{0}$ decreases again, whereas the amplification factor continues growing, in accordance to the convectively unstable nature of the $B L$ flow. Regarding the effect of increasing $D / \delta^{*}$ on $A(x) / A_{0}$ at a given location -e.g. at $x / \delta^{*}=120$, near the end of the groove- $A /\left.A_{0}\right|_{x / \delta^{*}=120}$ is up to 20 times larger than that corresponding to the unindented case, tables 2-3. Finally, observe how the $A(x) / A_{0}$ reaches the level $e^{8}$ up to $60 \delta^{*}$ units before the $F P$ for the higher $M a_{\infty}$.

350

Modes M3 (figures 10e-10f) behave slightly differently, as they show a differentiated behaviour with respect to $M a_{\infty}$ : whereas in both cases eigenmodes experience spatial amplification, an increase in indentation depth $D / \delta^{*}$ has a lasting effect only for $M a_{\infty}=0.1$ eigenmodes. $M a_{\infty}=0.5$ case modes see their amplification factor affected by $D / \delta^{*}$, but this effect disappears as $x / \delta^{*}$ increases. The $A(x) / A_{0}$ curves even collapse into each other beyond $x / \delta^{*} \approx 300$. Notice as well, how despite its relatively high temporal amplification factor $\omega_{I}$ (table 4 ), this mode does not grow significantly in space (note the scale range in figure 10f). 


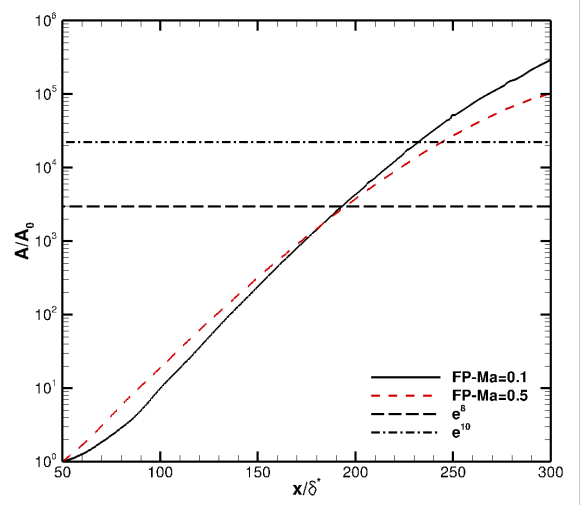

(a) FP

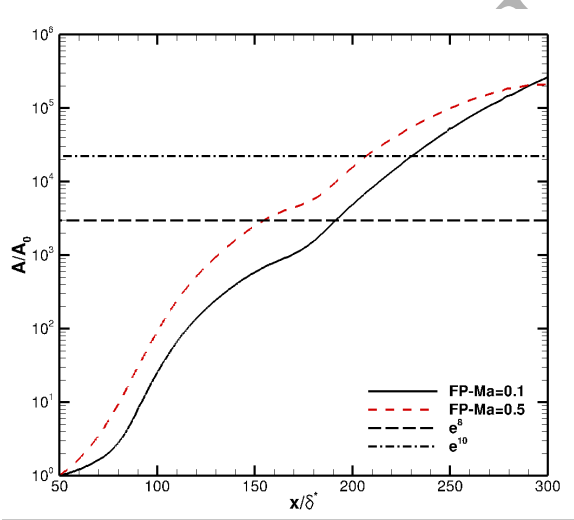

(b) $D / \delta^{*}=1$

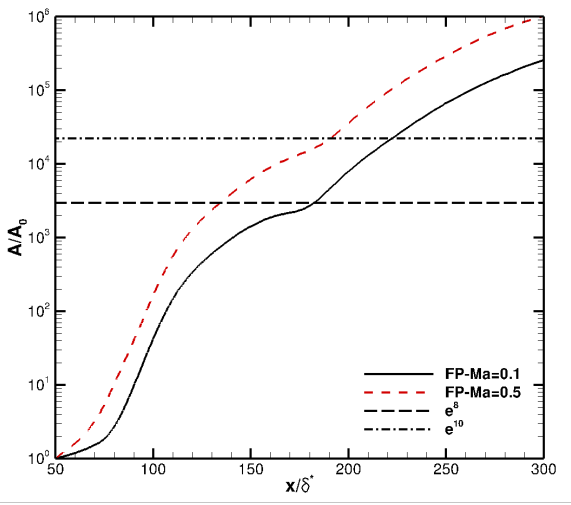

(c) $D / \delta^{*}=1.5$

Figure 9: Amplification factors for $M a_{\infty}=0.1$ ( - ) and $M a_{\infty}=0.5(---)$ for most spatially amplified mode $\left(\omega_{R} \approx 0.12-0.13\right)$. Horizontal lines run at levels $e^{8}(---)$ and $e^{10}$ $(-\cdot-\cdot-)$. 


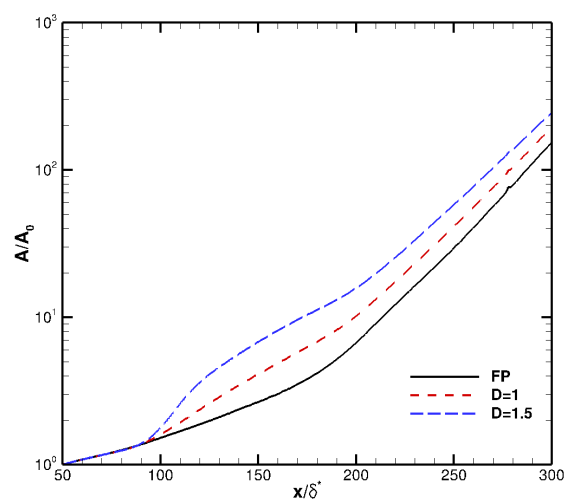

(a) M1 for $M a_{\infty}=0.1$

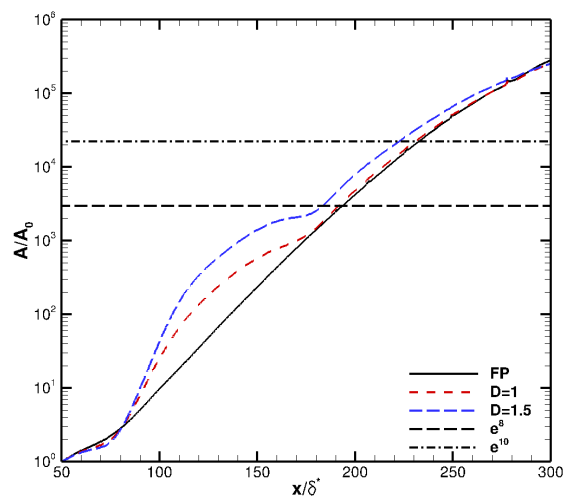

(c) M2 for $M a_{\infty}=0.1$

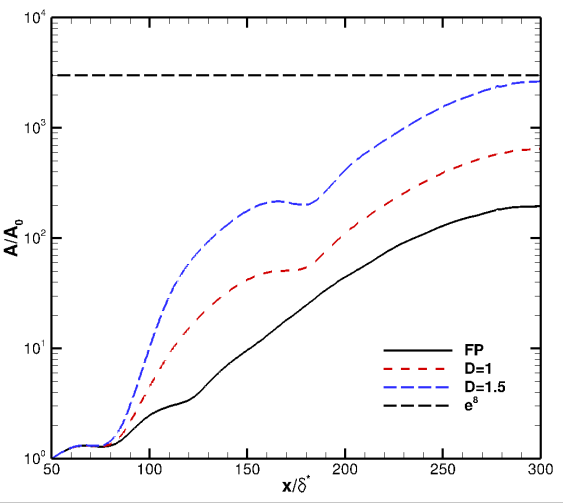

(e) M3 for $M a_{\infty}=0.1$

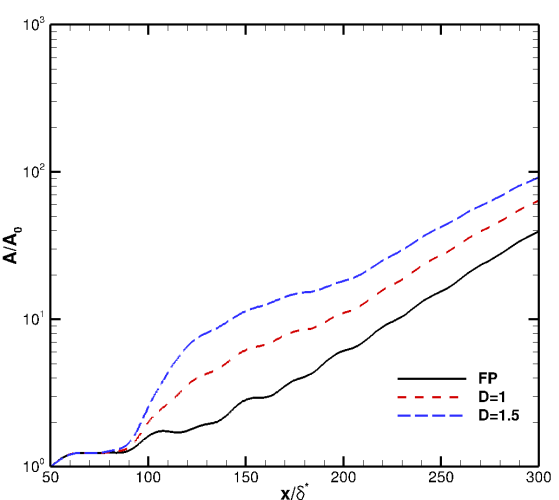

(b) M1 for $M a_{\infty}=0.5$

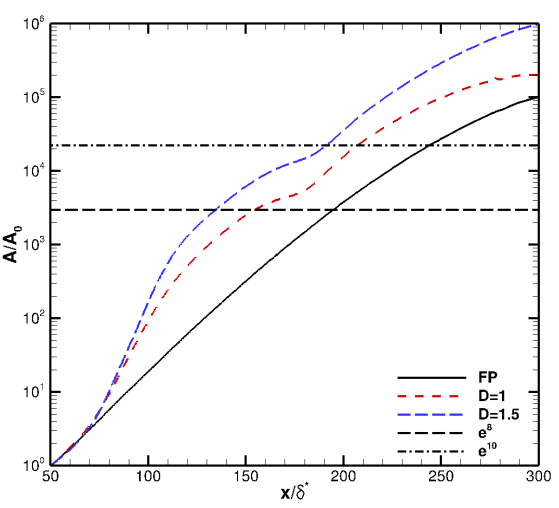

(d) M2 for $M a_{\infty}=0.5$

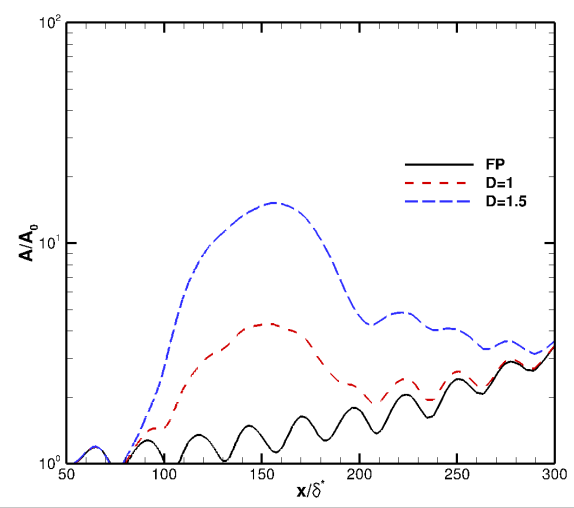

(f) M3 for $M a_{\infty}=0.5$

Figure 10: Amplification factors for FP (-), and depths $D / \delta^{*}=1\left({ }^{---}\right)$and $1.5(-\cdot-\cdot-)$.

Horizontal lines run at levels $e^{8}(---)$ and $e^{10}(-\cdot-\cdot-)$. 
A complementary vision of the effect of the indentation on the eigenfunctions can be gained by exploiting the concept of transmission coefficient, discussed in $[9,10]$. Consider the least temporally stable (M1) and the most spatially amplified (M2) modes for the $M a_{\infty}=0.5, D / \delta^{*}=1.5$ case. Curves (dashedblue ones in figure 11) fitting the high $x / \delta^{*}$ behaviour of those eigenfunctions to the spatial growth of the corresponding flat plate eigenmode have been obtained. These curves can be then traced back to the centre of the indentation $x / \delta^{*}=100$. This allows to estimate transmission coefficients of $A_{T} \approx 3$ and 10 for M1 and M2 modes, respectively. These values are consistent with/ those reported $[9,10]$. It should be noted that $\omega_{i}<0$ in our work, whereas $\omega$ is real (i.e. $\left.\omega_{i}=0\right)$ in the local scattering approach $[9,10]$. However, since $\omega \mathrm{i}$ is usually rather small, the transmission coefficient extracted here is likely to approximate that in $[9,10]$, and provides a measure of the destabilizing effect of the indentation.

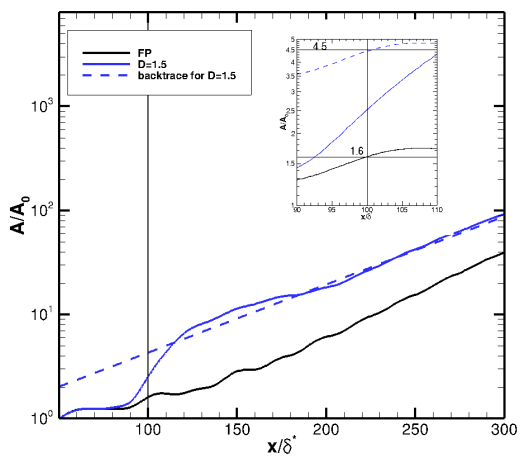

(a) M1

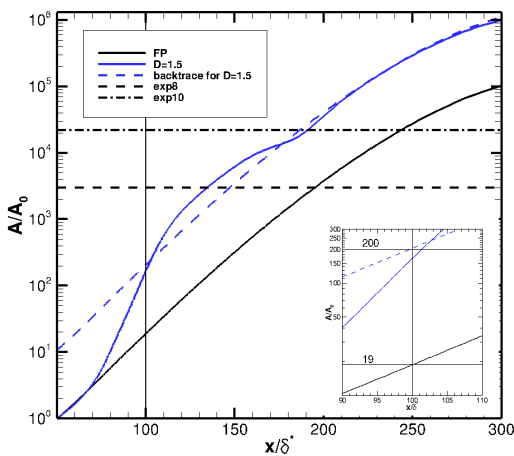

(b) M2.

Figure 11: Transmission coefficient estimation for M1 and M2 for the $M a_{\infty}=0.5, D / \delta^{*}=1.5$ case. 


\section{Conclusions}

Current wing manufacturing processes allow two-dimensional surface imperfections during the assembly phase. These imperfections may compromise the efficiency of natural laminar flow designs: since $N L F$ wings operate at Reynolds numbers in the unstable regime, the question arises whether these surface imperfections may advance laminar-to-turbulent transition via TS amplification. If that were effectively the case, the $N L F$ wing would be then operating suboptimally (increasing drag and fuel consumption).

In this contribution the influence of a groove on the stability characteristics of a flat plate boundary layer flow has been investigated. The indentation considered is representative of the smooth gap left by the retraction of a filler material applied at the junction of leading-edge and wing-box components.

Since the configuration considered is such that the parallel/weakly-parallel assumptions are necessarily compromised, we have performed a global temporal stability analysis. Specifically, our investigation has addressed the effect of increasing depth $D / \delta^{*}$ and Mach number $M a_{\infty}$ on perturbation time-decay rates and spatial amplification factors.

We have shown that temporal spectra are sensitive to both groove depth $D / \delta^{*}$ and upstream Mach number $M a_{\infty}$. While the Mach number effect is significant over the whole angular pulsation range, that of $D / \delta^{*}$ is relevant only in a part of that range.

400 At a given $M a_{\infty}$ number, an increase in groove depth translates into less temporally stable modes; for a given groove depth $D / \delta^{*}$, an increase in Mach number also renders modes less temporally stable. However, no matter the combination of parameters considered, the eigenvalues retrieved remain confined in the temporally stable region $\left(\right.$ i.e. $\left.\omega_{I}<0\right)$. 
We have also studied the impact of $M a_{\infty}$ and $D / \delta^{*}$ on the spatial amplification factors of the individual global eigenfunctions retrieved. As expected, not all the eigenmodes are equally processed by the indentation: while certain modes are slightly altered, others experience substantial modifications: the amplification at a given location can be up to 20 times larger than that corresponding to the unindented case. All in all, for a given angular pulsation ${ }^{2}$ an increase in $D / \delta^{*}$ results in a larger spatial amplification. An increase in $M a$ o may enhance further this behaviour.

415 In this context, one can find combinations of $\omega_{R}, D / \delta^{*}$ and $M \alpha_{\infty}$ that, according to an $e^{N}$ method, would lead to early transition soon after the indentation. This is more likely as $D / \delta^{*}$ and $M a_{\infty}$ increase, and poses concerns along two directions. On the one hand, it sets a limitation on the tolerances allowed when applying filler materials; on the other hand, an undesired premature transition might happen as flight $M a_{\infty}$ numbers are approached, invalidating the design effort invested in the $N L F$ wing.

Finally, care should be exerted when employing classical design tools relying on parallel or weakly non-parallel assumptions since indentations seem to enhance

\section{Acknowledgements}

This research has been funded through the NNATAC FP7 initiative (PIAP-GA-2012-324298).

This work has been fuelled by lively discussions with Dr. R. Ashworth (Airbus Group Innovations). The authors would like to thank the referees for their helpful comments and suggestions.

First author is grateful for Ms. E. Iorfida Gil's support.

\footnotetext{
${ }^{2}$ Included in the preferential angular pulsation range described already.
} 


\section{References}

[1] H. Reed, W. Saric, Stability of three-dimensional boundary layers, Annu. Rev. Fluid Mech. 21 (1989) 235-284. doi:10.1146/annurev.fl.21. 010189.001315

[2] R. Ashworth, S. Lawson, et. al., Numerical and experimental study of the tolerance of natural laminar flow on a wing to ts destabilisation at the leading edge /wing-box junction, Royal Aeronautical Society Applied Aerodynamics Conference, Bristol (UK) (2016).

[3] P. Ricco, J. Luo, X. Wu, Evolution and instability of unsteady nonlinear streaks generated by free-stream vortical disturbances, J. Fluid Mech. 677 (2011) 1-38.

[4] H. Schlichting, K. Gersten, E. Krause, H. Oertel, Boundary-layer theory, 8th Edition, Springer, Berlin, 2000.

445 [5] T. Herbert, Parabolized stability equations, Annu. Rev. Fluid Mech. 29 (1997) 245-283.

[6] J. van Ingen, A suggested semi-empirical method for the calculation of the boundary layer transition region, Tech. Rep. V.T.H. 74, TH Luftfahrtabteilung, Delft (1956).

[7] A. Smith, N. Gamberoni, Transition, pressure gradient and stability theory, TR ES-26388, Douglas Aircraft Company (1956).

[8] J. Zahn, U. Rist, Impact of deep gaps on laminarturbulent transition in compressible boundary-layer flow, AIAA Journal 54 (1) (2016) 66-76.

[9] Z. Huang, X. Wu, A local scattering approach for the effect of abrupt changes on boundary-layer instability and transition: a numerical method, in: Proceedings of the $45^{t h}$ AIAA Fluid Dynamics Conference, AIAA AVIATION Forum, AIAA, Dallas (TX), 2015. doi:http://dx.doi.org/10. 2514/6. 2015-2623. 
[10] X. Wu, M. Dong, A local scattering theory for the effects of isolated roughness on boundary-layer instability and transition: transmission coefficient as an eigenvalue, J. Fluid Mech. 794 (2016) 68 - 108. doi: $10.1017 / \mathrm{jfm} .2016 .125$.

[11] Z. Huang, X. Wu, A local scattering approach for the effects of abrupt changes on boundary-layer instability and transition: a finite-reynoldsnumber formulation for isolated distortions, J. Fluid Mech. 822 (2017) 444483. doi:10.1017/jfm.2017.287.

[12] V. Theofilis, Global linear instability, Annu. Rev. Fluid Mech. 43 (2011) 319-352.

[13] M. Marquillie, U. Ehrenstein, On the onset of nonlinear oscillations in a separating boundary-layer flow, J. Fluid Mech. 490 (2003) 169-188. doi: 10.1017/S0022112003005287.

[14] F. Gallaire, M. Marquillie, U. Ehrenstein, Three-dimensional transverse instabilities in detached boundary layers, J. Fluid Mech. 571 (2007) 221233. doi:doi:10.1017/S0022112006002898.

475 [15] U. Ehrenstein, F. Gallaire, Two-dimensional global low-frequency oscillations in a separating boundary-layer flow, J. Fluid Mech. 614 (2008) 315327. doi:10.1017/,S0022112008003285.

[16] E. Akervik, J. HCEpffner, U. Ehrenstein, D. Henningson, Optimal growth, model reduction and control in a separated boundary-layer flow using global eigenmodes, J. Fluid Mech. 579 (2007) 305-314. doi:10.1017/ so022112007005496.

[17] D. Rodriguez, V. Theofilis, Structural changes of laminar separation bubbles induced by global linear instability, J. Fluid Mech. 655 (2010) 280-305.

[18] H. Xu, S. Sherwin, P. Hall, X. Wu, The behaviour of Tollmien-Slichting 485 waves undergoing small-scale localised distortions, J. Fluid Mech. 792 (2016) 499-595. 
[19] U. Ehrenstein, F. Gallaire, On two-dimensional temporal modes in spatially evolving open flows: the flat-plate boundary layer, J. Fluid Mech. 536 (2005) 209-218. doi:10.1017/S0022112005005112.

[20] F. Alizard, J.-C. Robinet, Spatially convective global modes in a boundary layer, Phys. Fluids 19 (11) (2007) 114105. doi:10.1063/1.2804958,

[21] R. Ashworth, personal communication, November, 2015.

[22] C. Rowley, T. Colonius, A. Basu, On self-sustained oscillations in twodimensional compressible flow over rectangular cavities, J. Fluid Mech. 455 (2002) 315-346. doi:10.1017/S0022112001007534.

[23] G. Brès, T. Colonius, Three-dimensional instabilities in compressible flow over open cavities, J. Fluid Mech. 599 (2008) 309-339. doi:10.1017/ S0022112007009925.

[24] D. A. Kopriva, Multidomain Spectral Solution of Compressible Viscous Flows, J. Comput. Phys. 115

[25] D. Kopriva, A Staggered-Grid Multidomain Spectral Method for the Compressible Navier Stokes Equations, J. Comput. Physics 143 (1998) 125-158. doi: $10.1006 / j c p h .1998 .5956$.

[26] J. Wendt, J. Anderson, G. Degrez, Computational Fluid Dynamics. An Introduction, $3^{\text {rd }}$ Edition, Springer-Verlag Berlin Heidelberg, 2009.

[27] J. N. Lyness, C. B. Moler, Numerical differentiation of analytic functions, SIAM Journal for Numerical Analysis 4 (2) (1967) 202210.

[28] O. Browne, G. Rubio, E. Ferrer, E. Valero, Sensitivity analysis to unsteady perturbations of complex flows: a discrete approach, Int. J. Numer. Meth. Fluids 76 (2014) 1088-1110. doi:10.1002/fld.3962.

[29] E. Valero, E. Ferrer, J. de Vicente, Numerical Methods for Direct Numerical Simulation and Stability Analysis, in: E. Valero, F. Pinna (Eds.), Progress 
in Flow Instability Analysis and Laminar-Turbulent Transition Modeling, VKI Lecture Series 2014-05, Von Karman Institute for Fluid Dynamics, 2014.

[30] K. Meerbergen, A. Spence, D. Roose, Shift-invert and cayley transforms for detection of rightmost eigenvalues of nonsymmetric matrices, BIT $\mathrm{Nu}$ merical Mathematics 34 (1994) 409-423.

[31] Y. Saad, Iterative methods for sparse linear systems: Second edition, SIAM, 2003.

[32] P. Amestoy, A. Guermouche, J.-Y. LÉxcellent, S. Pralet, Hybrid scheduling for the parallel solution of linear systems, Parallel Computing 32 (2006) $136-156$.

[33] E. Åkervik, U. Ehrenstein, F. Gallaire, D. Henningson, Global two${ }_{525}$ dimensional stability measures of the flat plate boundary-layer flow, European Journal of Mechanics B/Fluids 27/(2008) 501-513. 